\title{
Controlled oil/water partitioning of hydrophobic substrates extends the bioanalytical applications of droplet-based microfluidics
}

\author{
Buryska Tomas $^{\dagger * \# \text {, Vasina Michal }}{ }^{\dagger * \#}$, Gielen Fabrice ${ }^{\S}$, Vanacek Pavel ${ }^{\dagger \star}$, van Vliet Liisa ${ }^{\S}$, Jezek Jan ${ }^{\perp}$, \\ Pilat Zdenek ${ }^{\perp}$, Zemanek Pavel ${ }^{\perp}$, Damborsky Jiri ${ }^{\dagger *}$, Hollfelder Florian ${ }^{\S}$, Prokop Zbynek ${ }^{\dagger * *}$ \\ \# These authors contributed equally to this work. \\ *Author for correspondence: Zbynek Prokop, zbynek@ chemi.muni.cz, +420 549496667. \\ $\dagger$ Loschmidt Laboratories, Department of Experimental Biology and RECETOX, Faculty of Science, Masaryk University, \\ Kamenice 5, 62500 Brno, Czech Republic \\ \$ International Clinical Research Center, St. Anne's University Hospital, Pekarska 53, 65691 Brno, Czech Republic \\ $\S$ Department of Biochemistry, University of Cambridge, 80 Tennis Court Road, Cambridge CB2 1GA, UK \\ || Living Systems Institute, University of Exeter, Exeter EX4 4QD, UK \\ $\perp$ Institute of Scientific Instruments, Czech Academy of Sciences, Kralovopolska 147, 61264 Brno, Czech Republic
}

\begin{abstract}
A number of novel protein sequences dramatically increases in public databases, however, there are only very few functional annotations. This significantly limits the exploration of the available protein diversity obtained by next-generation sequencing. Conventional methods cannot keep up with current challenges and a development of a new generation of biochemical techniques is essential. The great potential of microfluidic technology with reduced sample requirements and powerful throughput can bring an adequate capacity for functional annotation of the rising protein diversity. However, even the most promising dropletbased systems still need to address a number of limitations. The leakage of hydrophobic compounds from water compartments to the carrier oil represents one of the major problems which limits the utilization of these systems to operate with hydrophilic reagents. Herein, we present an approach using a novel way of substrate delivery in droplet microfluidics applied to high-throughput functional characterization of enzymes converting hydrophobic substrates. The substrate delivery is based on the partitioning of hydrophobic chemicals between the oil and water phases. We applied a controlled distribution of hydrophobic haloalkanes from oil to reaction water droplets to perform activity screening of eight model enzymes from the haloalkane dehalogenase family. The droplet-on-demand microfluidic system reduces the reaction volume 65000 times and increases the speed of the analysis almost 100 times, compared to the conventional method. Additionally, the microfluidic setup enables a convenient determination of temperature optima for a set of mesophilic and engineered hyper stable enzyme variants at the working range 5 to $90{ }^{\circ} \mathrm{C}$. A quantitative comparison of the microfluidic data and the results from the the conventional method showed a high consistency with $\mathrm{R}^{2}=0.89$ and 0.95 for the substrate specificity and temperature optima analysis, respectively. The microfluidic method demonstrated a high precision and an advanced analytical throughput $>20,000$ reactions per day. The presented substrate delivery approach extends the scope of microfluidics applications for high-throughput analysis of reactions including compounds with limited water solubility.
\end{abstract}

\section{INTRODUCTION}

Advances in sequencing technologies result in the accumulation of vast amounts of sequence information in majority cases without the knowledge of functional properties of the encoded proteins. However, a systematic functional characterization of multitude candidates cannot be addressed by conventional approaches even when automated liquid handling systems are applied. Microfluidic technology offers an appropriate throughput, reaching in extreme cases $10^{7}$ assays per day, and significant reduction for sample amount requirements. ${ }^{1}$ Droplet microfluidics has been employed in diverse fields from material chemistry synthesis of nanoparticles ${ }^{8}$ to life science rare cell isolation and sequencing. ${ }^{9,10}$ The ultrahigh-throughput has been exploited for example in on-chip sorting approaches for di- rected evolution of proteins is possible at $>\mathrm{kHz}$ rates in picolitre volumes. ${ }^{11-15}$ Amongst such droplet-based technologies, droplet-on-demand platforms enable the fast characterization of compound libraries and the acquisition of automated dose-response curves with exquisite control over droplet content and order. The droplets content can be analyzed by diverse analytical methods covering optical microscopy, electrical measurements, absorbance ${ }^{2}$ and fluorescence detection, ${ }^{3,4}$ Raman spectroscopy, ${ }^{5}$ mass spectroscopy ${ }^{6}$ or electrophoresis. ${ }^{7}$ Despite its great potential, droplet microfluidics still faces drawbacks like leakage of hydrophobic compounds, ${ }^{16}$ channel wetting and cross-contamination. ${ }^{17}$ The leakage of hydrophobic compounds (e.g. fluorophores, drugs/drug-leads, vitamins) from water compartments to the carrier oil represents one of the major problems and limits the use of these effective analytical systems. The process of extraction of hydrophobic molecules from the water 
droplet to the oil phase or neighbouring droplets can be as fast as a few milliseconds when convection plays a role. ${ }^{16,18}$ There is a number of attempts which, however, could only partially prevent the escape of hydrophobic compounds to the oil phase, e.g. addition of bovine serum albumin ${ }^{17}$ or sugar molecules, ${ }^{19}$ modifications of surfactant ${ }^{20}$ or using nanoparticles instead of surfactant. ${ }^{21}$ The leakage of hydrophobic compounds thus remains the major limitation of the droplet-based microfluidics.

Here we present a novel approach that addresses the control of hydrophobic compounds in the droplet microfluidic systems. The hydrophobic compounds pre-solubilized in the fluorinated oil phase are delivered to the dispersed aqueous phase by partitioning. At equilibrium, the final concentration of the compound in the water compartments was determined by its oil/buffer partitioning coefficient and the concentration of the compound in the oil phase. We demonstrated this approach during a biochemical characterization of a model enzyme family, haloalkane dehalogenases, converting a wide range of small hydrophobic halogenated alkanes. We determined the oil/buffer partitioning coefficients for series of 25 small mono-, di- and tri-halogenated aliphatic hydrocarbons. We used the oil/buffer distribution to control the delivery of these hydrophobic compounds into aqueous reaction droplets. A combinatorial analysis of the specific activity of 8 representative haloalkane dehalogenases with a set of 27 representative substrates (Supporting Figure S1) was performed within 24 hours, which represents a nearly 100 -fold reduction in time and a 10,000-fold lower requirement for the total amount of enzyme in comparison to a conventional assay. ${ }^{22}$ The substrate screening and additional analysis of the temperature optima for a set of mesophilic and hyper stable variants showed high consistency of the microfluidic data with the conventional measurements. We employed $\mathrm{pH}$-based fluorescence assays for monitoring the enzymatic activity in microfluidic droplets generally applicable for numerous enzyme families that are important for industrial or medical applications.

\section{MATERIALS AND METHODS}

Materials. All chemicals used in this study were research grade purity $>95 \%$ and were purchased from Merck (Merck, USA). The PicoSurf 1 surfactant was purchased from Dolomite (Dolomite, UK). FC40 oil was purchased from 3M (3M, USA). Tubing made from various materials was purchased from IDEX (IDEX, USA).

Oil/buffer partitioning of halogenated compounds. The partition coefficient of the tested halogenated compounds was analyzed by monitoring the distribution in a two-phase system composed by $1 \mathrm{~mL}$ HEPES buffer ( $1 \mathrm{mM}, \mathrm{pH} 8.0)$ and $1 \mathrm{~mL}$ FC40 oil (3M, USA). The analysis was performed in a screwcapped vial with a magnetic cap at $37{ }^{\circ} \mathrm{C}$. In both phases, the concentration of a particular compound was quantified using the gas chromatograph Trace 1300 (Thermo Scientific, USA) equipped with capillary column TG-SQC (30m x $0.25 \mathrm{~mm} \times$ $0.25 \mu \mathrm{m}$, Thermo Scientific, USA) and connected to the mass spectrometer ISQ LT Single Quadrupole (Thermo Scientific, USA). The $1 \mu \mathrm{L}$ of sample was injected into the split-splitless inlet at $250{ }^{\circ} \mathrm{C}$, with split ratio 1:50. The sample preparation was fully handled by an automatized robotic arm (Pal RTC, CTC analytics, Switzerland). The temperature program was isothermal at $40{ }^{\circ} \mathrm{C}$ for $1 \mathrm{~min}$, followed by an increase to $140{ }^{\circ} \mathrm{C}$ at $20{ }^{\circ} \mathrm{C} \cdot \mathrm{min}^{-1}$ and hold for $8 \mathrm{~min}$. The flow of carrier gas (He) was $1 \mathrm{~mL} \cdot \mathrm{min}^{-1}$. The spectrometer was operated at a SCAN mode (30 to $300 \mathrm{amu}$ ). The temperature of the ion source and GC-MS transfer line was $200{ }^{\circ} \mathrm{C}$ and $250-300{ }^{\circ} \mathrm{C}$, respectively. The partitioning coefficient was calculated as the logarithm of the compound concentration ratio in fluorinated oil and water using the following formula:

$$
\log P=\log \frac{\left[\text { compound }_{\text {oil }}\right]}{\left[\text { compound }_{\text {buffer }}\right]}
$$

\section{Substrate delivery and assay incubation}

A small incubation chamber for biochemical assay incubation consists of $20 \mathrm{~cm}$ capillary placed in a small glass vial. A substrate filled in a $1.5 \mathrm{~mL}$ glass vial with a gas-tight screw-capped lid penetrates via a polythene tubing (OD $0.4 \mathrm{~mm}$, ID $0.1 \mathrm{~mm}$, Smith-Medical, UK) to the oil phase and was finally delivered to the water droplet containing an enzyme sample. The incubation time can be regulated with flow rate from 1 to $5 \mathrm{~min}$. During the experiment, the vial was placed in Peltier element that enabled precise control of temperature (Meerstetter, Switzerland).

Droplet generation, signal acquisition and processing. Typically, $20 \mu \mathrm{L}$ of each enzyme sample were loaded into a 24 -well rack in the Dropix instrument (Dolomite, UK). An oil bath below the rack was prefilled with FC40 oil with $0.5 \%$ PicoSurf 1 surfactant. A fine bore polythene tubing (OD $0.4 \mathrm{~mm}$, ID $0.1 \mathrm{~mm}$, Smith-Medical, UK) was used for the droplet generation and the signal observation. Droplets were generated by a syringe pump (Chemyx, USA) running in the withdraw mode at a flow rate $10 \mu \mathrm{L} \cdot \mathrm{min}^{-1}$. Droplet volume, oil spacing and sample sequence were controlled using the Dropix control software. The tubing was perpendicularly aligned to an excitation/emission optical fiber within an in-house built black acetal cube. The excitation was achieved by a $450 \mathrm{~nm}$ blue laser $(12 \mathrm{~V}$, $200 \mathrm{~mW}$, PRC) focused by a spherical lens into the multimode optical fiber Y-bundle suitable for the reflection and backscatter spectroscopy (SQS, Czech Republic). The excitation wavelength was filtered on a dichroic mirror with the cut off at 490 $\mathrm{nm}$ (ThorLabs, Germany). The analogue signal was collected on an Si-detector (ThorLabs, Germany), converted to a digital signal by Ni-DAQ 6009 module (National Instruments, USA) and processed by LabView 12 (National Instruments, USA). The raw signal was processed by a droplet detection script written in MATLAB 2017b (Mathworks, USA). A brief description of the steps necessary to screen a single substrate is the following: loading of the solutions in the sample holder rack, starting of the pump, measurement of droplet data for calibration sequence, running and measurement of droplet trains containing the enzymatic samples separated by buffer droplets, stopping the flow and changing to the next substrate. The whole protocol took approximately 30 minutes for a single substrate for the eight enzymes tested. Signal analysis script is provided in the Supplementary material.

Robotic activity screening. Specific activities of LinB towards the set of 27 halogenated substrates were robotically analyzed using a Hamilton MICROLAB STARlet robot (Hamilton Robotics, Switzerland). The reactions were performed in $2 \mathrm{~mL}$ glass vials containing $1 \mathrm{~mL}$ of $100 \mathrm{mM}$ glycine buffer, $\mathrm{pH} 8.6$ and $1 \mu \mathrm{L}$ of the halogenated substrate at $37^{\circ} \mathrm{C}$. The reaction was initiated by an addition of the enzyme. The progress of the reaction was monitored by periodically withdrawing samples 
from the reaction mixture and immediately mixing these samples with $35 \%(\mathrm{v} / \mathrm{v})$ nitric acid to terminate the reaction. The release of the halide ion product was analyzed spectrophotometrically using the end-point assay developed by Iwasaki and coworkers. ${ }^{23}$ The dehalogenation activities were quantified as the rate of product formation over time.

Data analysis and statistics. A matrix of specific activities for the 8 HLDs and 27 halogenated substrates measured by the present method was constructed. The conventionally measured specific activities were taken from the work by Koudelakova $e t$ $a l . .^{22}$ Pearson and Spearman correlation coefficients were calculated for each substrate comparing conventional and capillary-based microfluidics data. The relationship between the enzymes and clustering to the substrate specificity groups was studied by Principal Component Analysis (PCA) using Statistica 13 (TIBCO, USA). A detailed explanation of the PCA was described previously. ${ }^{22}$ Briefly, the raw data were log-transformed and weighted relative to the individual enzyme's activity. Each value needed to be incremented by 1 to avoid the logarithm of zero values. The new values were then divided by the sum of values for a particular enzyme and weighted values were estimated. These transformed data were used to calculate principal components and the components explaining the highest variability in the data were then plotted for an identification of substrate specificity groups.

\section{RESULTS}

Design of microfluidic and optical platform. We designed the microfluidic platform combining the commercial robotic sampler Dropix (Dolomite Microfluidics, UK) and an in-house constructed incubation chamber, a temperature controller and an optical setup for monitoring the biochemical reactions in droplets. The microfluidic system employs a pump operatingin withdrawal mode to create droplets and load samples from the Dropix sampler into the capillary by using negative pressure. Sample order and the droplet volume was controlled by the Dropix sampler moving the capillary end horizontally and vertically. The droplets are generated by moving vertically the arm with a tubing end between oil and an aqueous sample. Access to up to 24 different samples is reached by horizontally positioning the arm along the rack with samples. Droplets are spaced by an oil phase continuously loaded from an oil bath. Generated droplet sequence travels through an incubation chamber filled with a particular halogenated compound. The compound penetrates through the capillary walls and equilibrates between the oil and aqueous droplets. An optical signal is detected with an optical fiber in a detection cube where an optical fiber coupled to a laser source is exciting the droplet and concurrently transfers the emitted light to the detector (Figure 1A).

During the development, it was necessary to find suitable capillary material meeting the following criteria: (i) minimal background signal for the fluorescence detection; (ii) permeability for halogenated substrates through the wall; (iii) relatively low thickness of the capillary wall; and (iv) inner and outer diameter dimensions compatible with the Dropix instrument. We have tested tubing made of polyethylene (PE), polyetheretherketone (PEEK), polytetrafluoroethylene (PTFE), fluorinated ethylene propylene (FEP), perfluoroalkoxy based (PFA) and Tygon. A very low or no substrate permeability was observed in the cases of FEP, PFA, PEEK and PTFE, therefore we did not use these tubing materials in the following experiments. Significant substrate concentrations in the oil and aqueous phases could be detected in the PE and Tygon tubing. We decided to use the PE tubing in all the following experiments as it had a thinner wall, lower background signal and simpler manipulation. In the setup we used, the PE tubing was submerged in the particular halogenated substrate in a glass vial going in and out through a septum with punched holes. Inside the vial, there was exactly 20 $\mathrm{cm}$ of the tubing bend in three round loops to prevent droplet squeezing and breakage (Supporting Figure S2). The transfer of the substrate can occur only in this looped region where the tubing was in direct contact with the halogenated substrate. The substrate passes the capillary wall barrier and then the equilibrium between the oil and aqueous phases is reached. The vials with the tubing immersed in the substrate were incubated for a minimum 12 hours prior to the examination. We have also developed an alternative approach suitable for the delivery of compounds not penetrating the tubing wall based on the addition of substrate-enriched oil to the primary oil flow (Supporting material).

Analysis of halogenated compounds partitioning and delivery. The equilibrium distribution between the fluorinated oil FC40 and a buffer solution (1 mM HEPES buffer, pH 8.0) was studied for a set of halogenated compounds by monitoring the concentration in both of the immiscible phases using gas chromatography (Supporting Table S1). The specific FC40/HEPES buffer partition coefficients ( $\log \mathrm{P}_{\mathrm{FC} / \text { buf }}$ ) ranged from -1.79 to 2.47 , however, most of the compounds prefer the fluorinated oil phase with $\log \mathrm{P}_{\mathrm{FC} / \mathrm{buf}}>0$. Partitioning coefficients $\log \mathrm{P}_{\mathrm{FC} / \text { buf }}$ correlates well with the partition coefficients for octanol/water $\left(\mathrm{LogP}_{\mathrm{oct} / \mathrm{wat}}\right)$ retrieved from the ChemSpider database $\left(\mathrm{R}^{2}=0.91\right)$ (Figure 2).

The relationship between $\log \mathrm{P}_{\mathrm{FC} / \text { buf }}$ and $\log \mathrm{P}_{\mathrm{oct} / \mathrm{wat}}$ indicates a reduced solubility of the tested compounds in FC40 in comparison to octanol which is consistent with previous studies assuming reduced solubility of short halogenated compounds in fluorinated oils. ${ }^{24,25}$ Still, the concentrations reached several to ten's $\mathrm{mM}$ in fluorinated oil and thanks to lowering of $\log \mathrm{P}_{\mathrm{FC} / \mathrm{buf}}$ all the tested compounds approached a concentration in water phase comparable with conditions achieved in a conventional assay where the compounds are solubilized directly in an aqueous phase. The relationship between $\log \mathrm{P}_{\mathrm{FC} / \text { buf }}$ and $\log$ $\mathrm{P}_{\text {oct/wat }}$ was used for an estimation of an equilibrium distribution of the tested compound in a two-phase microfluidic system but can also be applied for a prediction of the behavior of other structurally similar compounds. Next, we estimated the kinetics of the distribution process. We calculated the diffusion times of 1,2-dibromoethane in $\mathrm{H}_{2} \mathrm{O}$ and $\mathrm{FC} 40$ for a distance of $1 \mu \mathrm{m}$ under static conditions using the methodology described previously elsewhere (Supporting Material). ${ }^{16}$ The calculated times $0.61 \mathrm{~ms}$ and $1.51 \mathrm{~ms}$ for $\mathrm{H}_{2} \mathrm{O}$ and $\mathrm{FC} 40$, respectively, indicate rapid equilibration during compound delivery with no significant limitations for the kinetics of the biochemical reaction, which incubate on a time scale of minutes. The compound transfer and equilibration is expected to be even faster during the flow regime of operation due to convection. ${ }^{26}$ The transfer of the compounds to the oil and water phase has been tested experimentally in a microfluidic system (Supporting Figure S2). 


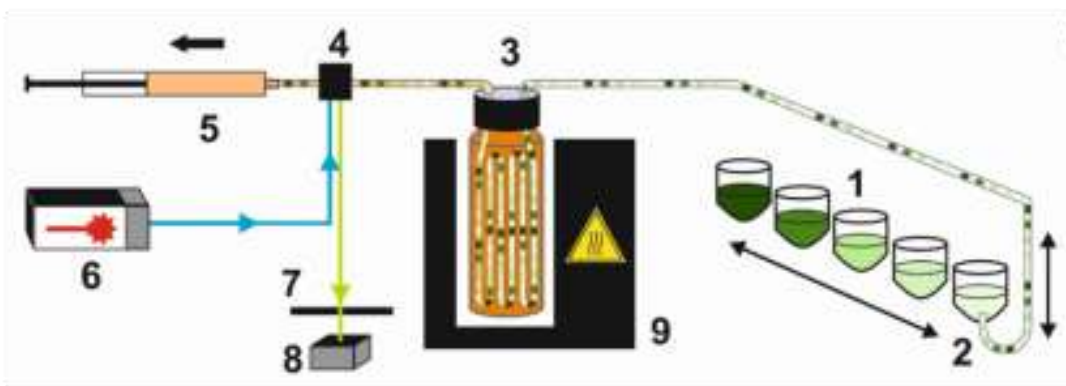

B
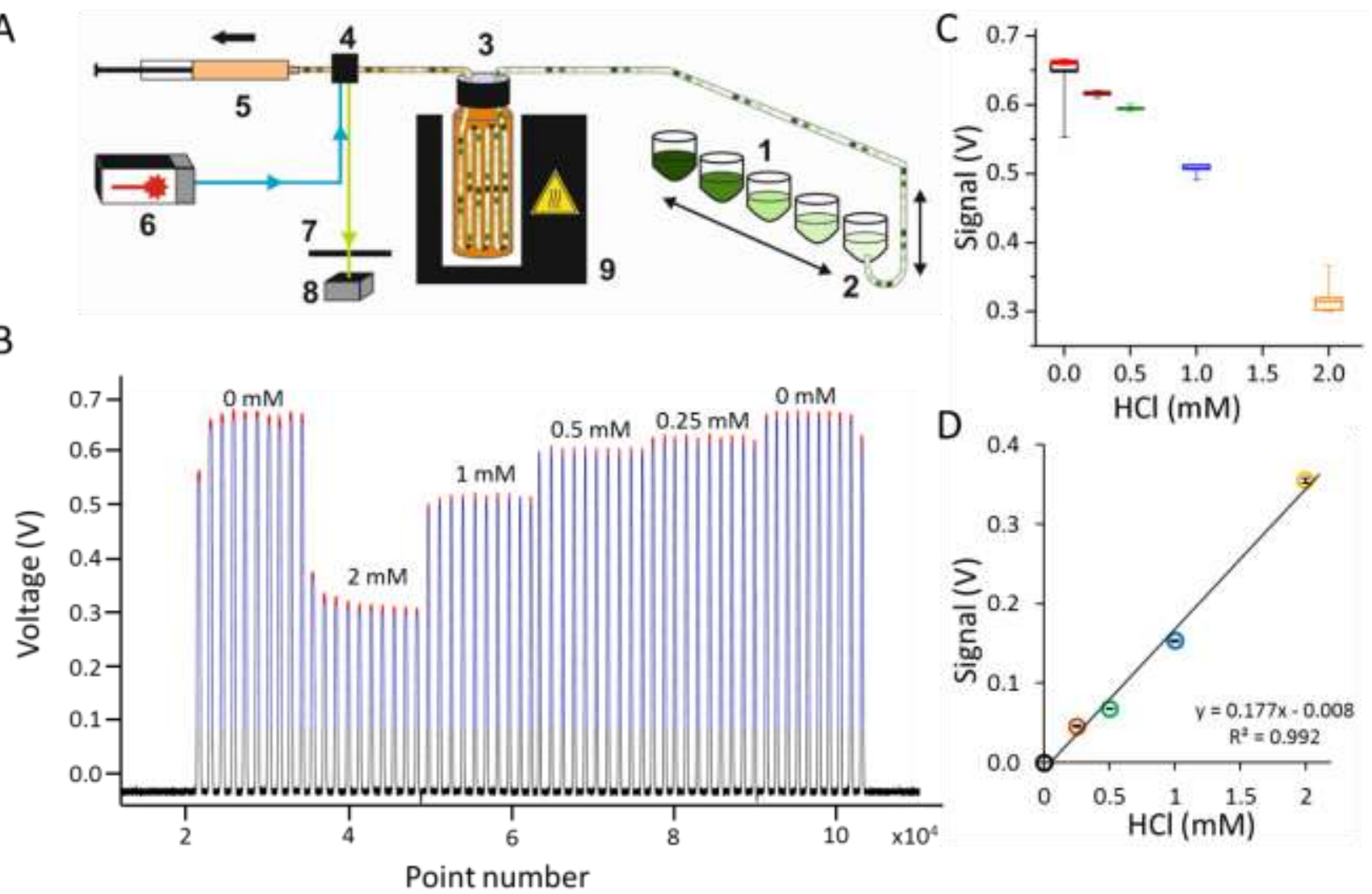

Figure 1: Capillary-based microfluidic platform and its calibration. (A) A scheme of the platform. Aqueous samples are loaded in a bottomless rack (1), which is placed at the top of an oil bath of the Dropix instrument. Droplets are generated by a vertical movement of a hook (2) with a polyethene tubing up and down between the oil bath and the rack with samples. Different enzyme samples are exchanged by a horizontal movement of the hook. Polyethene tubing connected to a syringe pump in a withdrawal mode (5) is going through an incubation chamber (3) and a black Delrin (DuPont) cube (4) serving as a detection point. Excitation light from a laser source (6) is brought to the tubing inside the detection cube. The reflected emission light is collected after passing through a dichroic mirror (7) at a photodetector (8). The system temperature is precisely controlled by a custom-made heating block (9). (B) A raw signal from the calibration sequence. Dilution series of a hydrochloric acid run in ten repetitions was used in the calibration. The blue color represents a signal above the threshold used for the droplet detection. The red color represents a signal peak averaged during the droplet analysis. (C) The mean value of the measured signal for each concentration. A box represents $25 / 75$ percentile and whiskers minimum/maximum of the data. The initial and ending droplet buffer sequences are black and red, respectively. (D) A linear regression was used to obtain a calibration slope. The error bars represent standard error to the data.

We studied the concentration of compounds in FC40 and aqueous droplets and the dependency on varying the aspiration rate from $5 \mu \mathrm{L} \cdot \mathrm{min}^{-1}$ to $20 \mu \mathrm{L} \cdot \mathrm{min}^{-1}$ that resulted in droplet residence times from 1 to 5 minutes. The different flow rate (different incubation time) did not significantly affect the final concentration of the compounds in both phases supporting the rapid equilibration estimated from diffusion times. The concentrations for the tested compounds ranged based on solubility from 3 to 60 $\mathrm{mM}$ in the FC40 and from 0.4 to $20 \mathrm{mM}$ in the buffer.

Design of temperature control. To control the temperature of the incubation chamber, we manufactured a copper block with a drilled hole for bringing a thermocouple into direct contact with the vial (Supporting Figure S3). Heating and cooling were achieved by a Peltier element glued to the copper block and controlled by the manufacturer's computer software. Excess heat was removed by a heat pipe connected to a cooler with a fan. The system working temperature ranged from $5{ }^{\circ} \mathrm{C}$ to 90
${ }^{\circ} \mathrm{C}$, with $0.1{ }^{\circ} \mathrm{C}$ accuracy. Heat transfer in the droplets on a short distance is a fast phenomenon and the droplet reaches target temperature upon arrival to the vial placed in the heated block in less than $1 \mathrm{~s}^{8}{ }^{8}$

Design of detection method. We employed pH-based fluorescence assays for monitoring the enzymatic activity in the microfluidic droplets generally applicable for numerous enzyme families that are important for industrial or medical applications (e.g., esterases, ureases, lipases, proteases, pyruvate kinases, glycoside hydrolases or glucose oxidases). Small changes in the $\mathrm{pH}$ can be observed in simple systems consisting of a weak buffer, e.g. HEPES, and complementary fluorescent indicator, e.g. 8-hydroxypyrene-1,3,6-trisulfonic acid (HPTS). ${ }^{27}$ The reaction mixture acidification results in a decrease of the fluorescence signal that is monitored with a planar laser-induced fluorescence by a single optical fibre. ${ }^{28}$ 


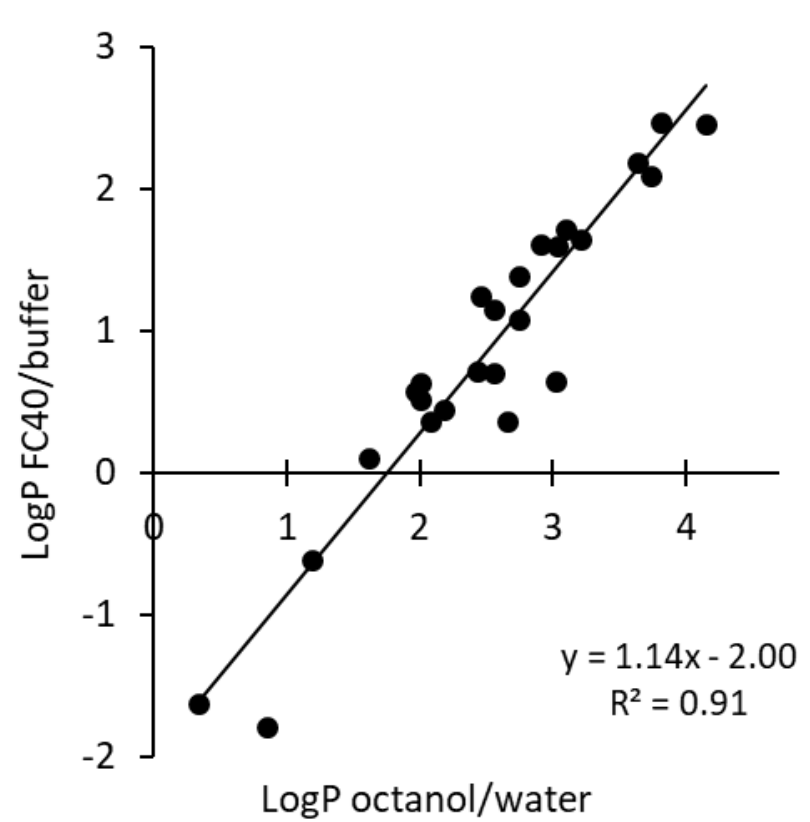

Figure 2: Comparison of partitioning coefficients. Correlation between the partitioning coefficients of 25 halogenated compounds obtained in octanol/water and in FC40/HEPES buffer at $37{ }^{\circ} \mathrm{C}$. The octanol/water partitioning coefficients were retrieved from the ChemSpider web server (http://www.chemspider.com). Partitioning in the fluorinated oil FC40 and aqueous 1mM HEPES buffer (pH 8.0) was determined using a gas chromatography as described in the Methods section.

A reaction progress as an end-point measurement is recorded after passing through the incubation chamber inside a detection cube bringing the tubing and the optical fiber to a close proximity at a perpendicular orientation (Figure 2A). Planar laser-induced fluorescence approach was used for the signal acquisition. ${ }^{28}$ Blue laser $(450 \mathrm{~nm})$ was focused into the optical fiber, thus directly the exciting content of the tubing. The perpendicular position of the optical fiber and the tubing was secured by an in-house fabricated black acetal cube with a screw-thread for the fiber. The excitation light is transferred to the sample only in the central part of the optical fiber, whereas the emission light was collected by all surrounding fibers. Such an optical bundle setup significantly reduces noise from the excitation light and increases the amount of the collected light where the excitation source has enough power. The excitation wavelength was filtered using a dichroic mirror with the cut-off $490 \mathrm{~nm}$. Finally, an analogue-to-digital board was used to read the voltage output from the photodetector. The signal was recorded using our own LabView code and further processed in MATLAB. The assembled system was calibrated for the monitoring of the $\mathrm{pH}$ change using $2 \mathrm{mM}, 1.0 \mathrm{mM}, 0.5 \mathrm{mM}$ and $0.25 \mathrm{mM} \mathrm{HCl}$.. The calibration was performed in the HEPES buffer ( $1 \mathrm{mM}$ HEPES, $20 \mathrm{mM} \mathrm{Na}_{2} \mathrm{SO}_{4}, \mathrm{pH}$ 8.0) with $50 \mu \mathrm{M}$ HPTS as the fluorescence indicator. A further decrease in the acid concentration was not distinguishable from the buffer solution (Figure 1B). The calibration consisted of a continuous sequence of the buffer, $\mathrm{HCl} / \mathrm{HBr}$ in a descending concentration and a buffer, where each solution was loaded as 10 droplets of $150 \mathrm{~nL}$ volume with
$300 \mathrm{~nL}$ oil spacing. Such a calibration sequence was repeated every time a new tubing was inserted in the reading cube to estimate the calibration slope. The standard deviation among droplets with the same solution was typically lower than $1 \%$ (Figure 1).

Comparison of microfluidic, robotic and conventional method. Specific activities of the model enzyme, haloalkane dehalogenase LinB from Sphingobium japonicum UT26, ${ }^{29}$ with a standard set of 27 representative substrates were measured using the microfluidic system and a liquid handling robot. Both the microfluidic and robotic data sets were compared with previously reported values obtained by conventional biochemical assay (Supporting Table S2). ${ }^{22}$ A positive enzymatic activity was observed for 23 substrates, while 4 substrates were not converted. This result was consistent throughout all the three data sets, conventional, robotic and microfluidic (Figure 3A). A quantitative analysis showed a strong correlation of the conventional measurement with robotic and microfluidic analysis with the Pearson coefficients 0.89 and 0.94, respectively (Figure 3A inset). A slight shift was observed for absolute values, the robotic data exhibit a $23 \%$ increase while microfluidic data a 37 $\%$ decrease of averaged activity values in comparison to the conventionally measured data set. Generally, the conventional method requires about 2-3 weeks to measure the substrate specificity profile for a single enzyme. The employment of robotic liquid handling sped up the process about 8 -fold. ${ }^{30}$ However, it still required relatively large amounts of samples (Table 1). The microfluidic screening of 8 model enzymes with 27 substrates was achieved within 24 hours, which speeds up the process nearly 100 times in comparison to the conventional method. The reaction volume scaled down to the $150 \mathrm{~nL}$, representing about a 65000 -fold reduction in the sample volume requirement.

Table 1: Comparison of the conventional, robotic and capillary-based microfluidic determination of the substrate specificity profiles.

\section{Conventional Robotic Microfluidic}

\begin{tabular}{l|ccc}
\hline $\begin{array}{l}\text { Reaction volume } \\
(\mathrm{mL})\end{array}$ & 10 & 1 & 0.00015 \\
$\begin{array}{l}\text { Total enzyme } \\
(\mathrm{mg})\end{array}$ & 540 & 54 & 0.05 \\
Total time (days) & 100 & 30 & 1 \\
& + & ++ & +++ \\
Reproducibility & +++ & -- & -- \\
Complexity & & $\$ \$ \$$ & $\$$ \\
& $\$$ & & \\
\hline
\end{tabular}




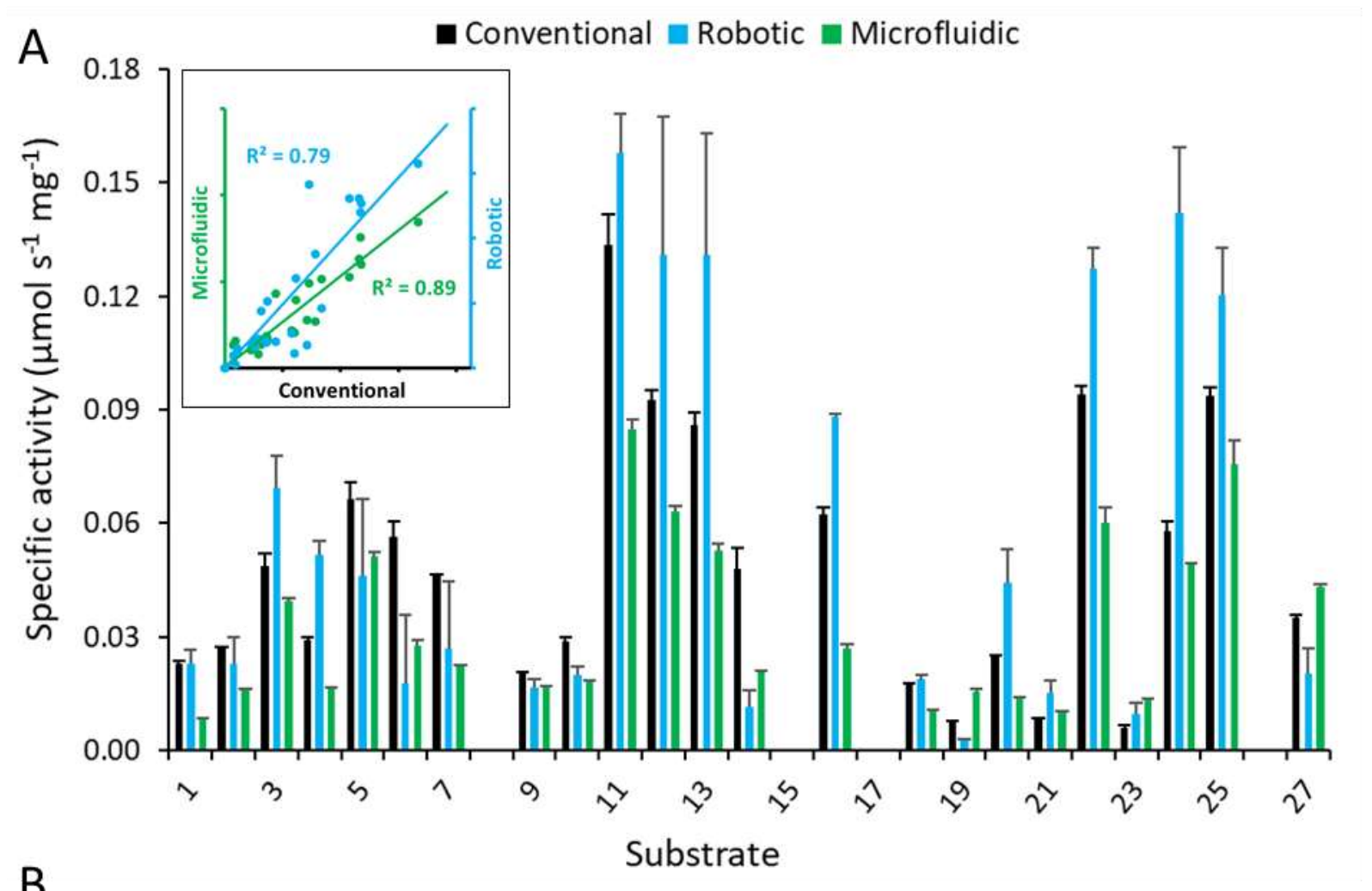

B
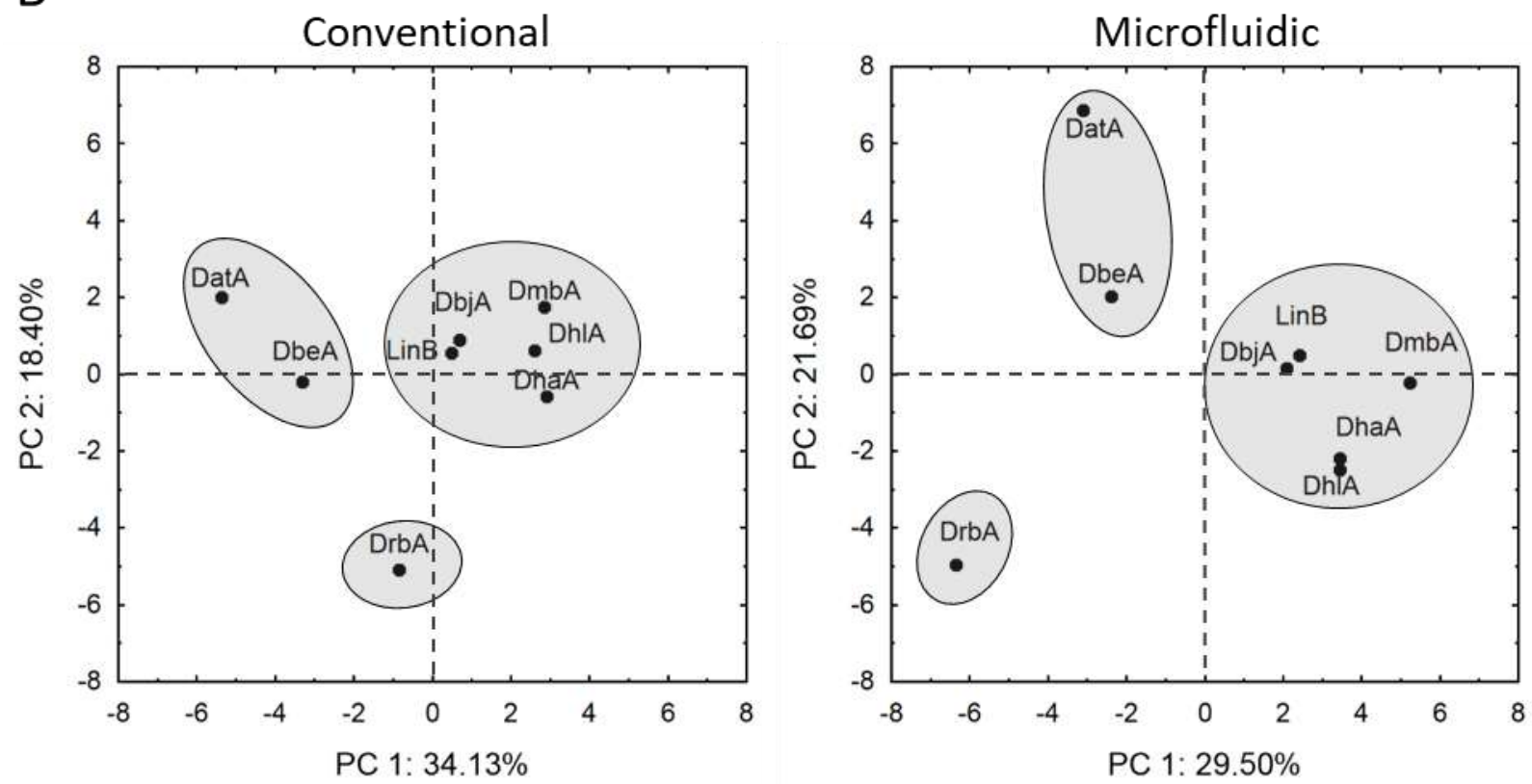

Figure 3: Analysis of substrate specificity and Principal Component Analysis. (A) Comparison of the substrate specificity profiles of HLD LinB determined by using a conventional method (black), a liquid handling robot (green) and a capillary-based microfluidics (orange). A positive activity for 23 out of 27 tested substrates was observed in all cases. A complete list of substrates and their specific activities is provided in the Supplementary material. The error bars represent standard errors to the data. Specific activities for the conventional measurement were taken from Koudelakova et al. ${ }^{22}$ The inset shows the correlation for the specific activities of the conventional, robotic (green) and capillary-based microfluidics data (orange). (B) Comparison of the specificity profiles of 8 different enzymes using Principal Component Analysis of the transformed specific activities determined with 27 halogenated compounds. The score plots presenting the data from the conventional and the capillary-based microfluidics. The score plots are two-dimensional projections of two factors covering the highest variability of the data. HLDs cluster into three substrate specificity groups visualized using the ovals. 
Validation of microfluidic method in combinatorial specificity screening of eight model enzymes. Next, we applied the microfluidic system for combinatorial screening of the activity of eight HLDs towards a set of 27 representative substrates (Supporting Table S3). The obtained microfluidic data were statistically analyzed by using Principal Component Analysis (PCA) and compared with conventional data reported previously by Koudelakova et al. (Figure 3B). ${ }^{22}$ The PCA analysis of both the conventional and microfluidic analyses identically clustered the enzymes to three substrate specificity groups. The Principal components 1 and 2 summed cover 52.4 and $51.1 \%$ variances in the data for the conventional and microfluidic approaches, respectively. Both analyses yield closely similar coordinates for the same enzymes. Larger deviations were detected only for the DrbA and DatA, which both represent enzymes with lower specific activities.

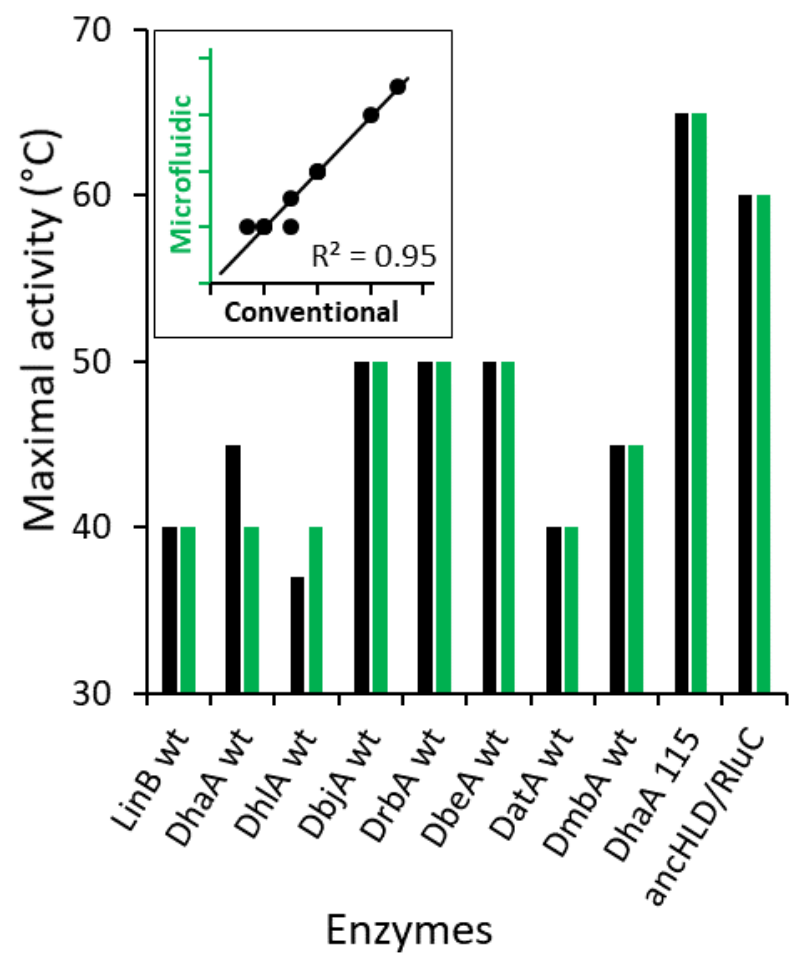

Figure 4: Evaluation of temperature optima. Comparison of enzyme temperature optima determined by a conventional method (white) and capillary-based microfluidics (black). Conventionally determined temperature optima were extracted from the study of Koudelakova et al. ${ }^{31}$ LinB, DmbA, DhlA, DbeA, DatA, DrbA, DbjA, and DhaA the wild type enzymes, DhaA 115 is a hyperstable variant engineered by computational protein design $^{32}$ and ancHLD-RLuC is a hyperstable reconstructed ancestral protein of HLDs and Renilla luciferase. The inset shows the correlation between conventional and microfluidic data.

Analysis of temperature optima. The temperature optimum was determined for the set of eight HLDs previously used for the specificity screening, all showing mesophilic characteristic with temperature optimum between $40{ }^{\circ} \mathrm{C}$ to $50{ }^{\circ} \mathrm{C}$. ${ }^{31}$ For better comparison, the set of tested enzymes was additionally enriched by two HLDs variants with significantly improved thermal stability: (i) hyperstableDhaA115 engineered by using FireProt computational protein design ${ }^{32}$ and (ii) hyperstable ancHLDRLuc, which is a reconstructed ancestral protein of HLDs and Renilla luciferase (unpublished results) (Figure 4). In the experiment, 1,2-dibromoethane served as a substrate and the reactions were measured by the 5-degree increment from $30^{\circ} \mathrm{C}$ to $80{ }^{\circ} \mathrm{C}$. The temperature optima obtained by microfluidic showed an excellent correlation $\left(\mathrm{R}^{2}=0.95\right)$ with conventional data previously reported for the tested enzyme variants (Figure 4 inset).

\section{CONCLUSIONS}

In the classical droplet microfluidics, a droplet is often considered as a self-contained reactor flowing in the carrier phase. ${ }^{26}$ However, droplets are not isolated objects and a leakage of hydrophobic compounds to an oil phase can be only partially prevented. ${ }^{20,33}$ We have shown that hydrophobic compounds can be distributed from an oil phase (substrate stock) into an aqueous droplets while keeping the substrate saturating conditions for the maximum activity performance. This overcomes the problem of containment of hydrophobic compounds in droplets in the classical droplet microfluidics, where compounds with an octanol-water partition coefficient larger than zero escape from water reaction droplets and disperse in fluorinated oil. Instead of treating droplets as isolated compartments in the carrier phase, we deliver hydrophobic compounds via a water-oil interface by the means of reaching equilibrium between the two phases. Substrate loading via an oil phase enables an analysis of hydrophobic compounds, which would not be possible using the classical approach where all reactants are present and delivered via aqueous solutions. Our analysis of short halocarbons revealed a high correlation $\left(\mathrm{R}^{2}=0.91\right)$ of water-oil partitioning with the $\log$ Ps reported in standard octanol-water conditions.

The novel substrate delivery opened the possibility to adopt classical microfluidics for the analysis of reactions including compounds with limited water solubility. We demonstrated the utility of this approach during the combinatorial screening of the activity of the model enzymes family, haloalkane dehalogenases, with a representative set of hydrophobic substrates. The biochemical data obtained using the droplet-based microfluidic system were critically compared with the conventional and robotic analyses. The quantitative comparison showed a high correlation between conventional and microfluidic measurements with the correlation coefficients 0.94 and 0.98 for substrate specificity and temperature optimum data, respectively. The presented platform will find use in screening campaigns for rapid profiling of activity, substrate specificity and temperature profiles from psychrophilic to thermophilic enzymes. The presented method uses a fluorescence probe to monitor a change of $\mathrm{pH}$ universal for numerous enzyme families that are important for industrial or medical applications (e.g., carboxylesterases, acetylcholinesterases, ureases, lipases, proteases, pyruvate kinases, glycoside hydrolases or glucose oxidases), without requirements for specific fluorogenic substrates. Additionally, the presented detection approach is not limited to the fluorescence only and can easily be applied also in the absorbance mode as demonstrated elsewhere ${ }^{34-36}$. 


\section{ASSOCIATED CONTENT}

\section{Supporting Information}

Technical details on the alternative substrate delivery process are described. Data used in the estimation of the partitioning coefficients. Concentrations of selected substrates in aqueous and oil phase are provided. The table used for comparison of raw specific activities on LinB. Raw data used in the statistical analyses. Raw signal analysis script for extraction of droplet signal in MATLAB is also provided.

\section{AUTHOR INFORMATION}

\section{Corresponding Author}

* Zbynek Prokop, zbynek@ chemi.muni.cz, +420 549496667.

\section{Author Contributions}

TB designed and conducted the experiments, analyzed and interpreted the data and wrote the manuscript. MV designed and conducted the experiments, analyzed the data and wrote the manuscript. FG and LV designed the experiments. PV conducted experiments and interpreted the data. JJ, ZPi and PZ contributed to the system development. FH and JD wrote the manuscript. ZPr designed the microfluidics setups and experiments, interpreted the data and wrote the manuscript.

\section{ACKNOWLEDGEMENT}

This research is supported by the Czech Science Foundation (GA16-07965S), infrastructure was supported by the Ministry of Education Youths and Sports of the Czech Republic (LQ1605 LO1214, CZ.02.1.01/0.0/0.0/16_026/0008451, CZ.02.1.01/0.0/0.0/16_019/0000868, LM2015051, CZ.02.1.01/0.0/0.0/16_013/0001761) and the European Commission (720776, CZ.1.05/2.1.00/01.0017). We acknowledge Zdenek Janicek for the help with acquisition of the robotic data and David Damborsky for his help with the estimation of partitioning coefficients.

\section{REFERENCES}

(1) Dittrich, P. S.; Manz, A. Lab-on-a-Chip: Microfluidics in Drug Discovery. Nat. Rev. Drug Discov. 2006, 5 (3), 210-218. https://doi.org/10.1038/nrd1985.

Gielen, F.; Hours, R.; Emond, S.; Fischlechner, M.; Schell, U.; Hollfelder, F. Ultrahigh-Throughput-Directed Enzyme Evolution by Absorbance-Activated Droplet Sorting (AADS). Proc. Natl. Acad. Sci. U. S. A. 2016, 113 (47), E7383-E7389. https://doi.org/10.1073/pnas.1606927113.

(3) Olguin, L. F.; Askew, S. E.; O’Donoghue, A. C.; Hollfelder, F. Efficient Catalytic Promiscuity in an Enzyme Superfamily: An Arylsulfatase Shows a Rate Acceleration of 10(13) for Phosphate Monoester Hydrolysis. J. Am. Chem. Soc. 2008, 130 (49), 16547 16555. https://doi.org/10.1021/ja8047943.

(4) Clausell-Tormos, J.; Lieber, D.; Baret, J.-C.; El-Harrak, A.; Miller, O. J.; Frenz, L.; Blouwolff, J.; Humphry, K. J.; Köster, S.; Duan, H.; et al. Droplet-Based Microfluidic Platforms for the Encapsulation and Screening of Mammalian Cells and Multicellular Organisms. Chem. Biol. 2008, 15 (5), 427-437. https://doi.org/10.1016/j.chembiol.2008.04.004.

(5) Kim, H. S.; Waqued, S. C.; Nodurft, D. T.; Devarenne, T. P.; Yakovlev, V. V; Han, A. Raman Spectroscopy Compatible PDMS Droplet Microfluidic Culture and Analysis Platform towards On-Chip Lipidomics. Analyst 2017, 142 (7), 1054-1060. https://doi.org/10.1039/c6an02221a.
Fidalgo, L.; Whyte, G. Coupling Microdroplet Microreactors with Mass Spectrometry: Reading the Contents of Single Droplets Online. Angew. Chemie Int. Ed. 2009, 3665-3668. https://doi.org/10.1002/anie.200806103.

(7) Roper, M. G.; Shackman, J. G.; Dahlgren, G. M.; Kennedy, R. T. Microfluidic Chip for Continuous Monitoring of Hormone Secretion from Live Cells Using an Electrophoresis-Based Immunoassay. Anal. Chem. 2003, 75 (18), 4711-4717. https://doi.org/10.1021/AC0346813.

Lignos, I.; Stavrakis, S.; Nedelcu, G.; Protesescu, L.; deMello, A J.; Kovalenko, M. V. Synthesis of Cesium Lead Halide Perovskite Nanocrystals in a Droplet-Based Microfluidic Platform: Fast Parametric Space Mapping. Nano Lett. 2016, 16 (3), 1869-1877. https://doi.org/10.1021/acs.nanolett.5b04981.

(9) Klein, A. M.; Mazutis, L.; Akartuna, I.; Tallapragada, N.; Veres, A.; Li, V.; Peshkin, L.; Weitz, D. A.; Kirschner, M. W. Droplet Barcoding for Single-Cell Transcriptomics Applied to Embryonic Stem Cells. Cell 2015, 161 (5), 1187-1201. https://doi.org/10.1016/j.cell.2015.04.044.

(10) Zilionis, R.; Nainys, J.; Veres, A.; Savova, V.; Zemmour, D.; Klein, A. M.; Mazutis, L. Single-Cell Barcoding and Sequencing Using Droplet Microfluidics. Nat. Protoc. 2016, 12 (1). https://doi.org/10.1038/nprot.2016.154.

(11) Agresti, J. J.; Antipov, E.; Abate, A. R.; Ahn, K.; Rowat, A. C.; Baret, J.; Marquez, M.; Klibanov, A. M.; Griffiths, A. D.; Weitz, D. a. Ultrahigh-Throughput Screening in Drop-Based Microfluidics for Directed Evolution. Proc. Natl. Acad. Sci. U. S. $\begin{array}{llll}\text { A. 2010, } & 107 & \text { (9), } & \text { 4004-4009. }\end{array}$ https://doi.org/10.1073/pnas.0910781107.

(12) Kintses, B.; Hein, C.; Mohamed, M. F.; Fischlechner, M.; Courtois, F.; Lainé, C.; Hollfelder, F. Picoliter Cell Lysate Assays in Microfluidic Droplet Compartments for Directed Enzyme Evolution. Chem. Biol. 2012, 19 (8), 1001-1009. https://doi.org/10.1016/j.chembiol.2012.06.009.

(13) Colin, P.-Y.; Kintses, B.; Gielen, F.; Miton, C. M.; Fischer, G.; Mohamed, M. F.; Hyvönen, M.; Morgavi, D. P.; Janssen, D. B.; Hollfelder, F. Ultrahigh-Throughput Discovery of Promiscuous Enzymes by Picodroplet Functional Metagenomics. Nat. Coтmun. 2015, 6, 10008. https://doi.org/10.1038/ncomms10008.

(14) Mair, P.; Gielen, F.; Hollfelder, F. Exploring Sequence Space in Search of Functional Enzymes Using Microfluidic Droplets. Curr. Opin. Chem. Biol. 2017, 37, 137-144. https://doi.org/10.1016/j.cbpa.2017.02.018.

(15) Obexer, R.; Godina, A.; Garrabou, X.; Mittl, P. R. E.; Baker, D.; Griffiths, A. D.; Hilvert, D. Emergence of a Catalytic Tetrad during Evolution of a Highly Active Artificial Aldolase. Nat. Chem. 2016, 9 (August), 50-56. https://doi.org/10.1038/nchem.2596.

(16) Chen, Y.; Wijaya Gani, A.; Tang, S. K. Y. Characterization of Sensitivity and Specificity in Leaky Droplet-Based Assays. Lab $\begin{array}{lllll}\text { Chip } & \text { 2012, } & 12 & \text { (23), } & \text { 5093-5103. }\end{array}$ https://doi.org/10.1039/c2lc40624a.

(17) Courtois, F.; Olguin, L. F.; Whyte, G.; Theberge, A. B.; Huck, W. T. S.; Hollfelder, F.; Abell, C. Controlling the Retention of Small Molecules in Emulsion Microdroplets for Use in Cell-Based Assays. Anal. Chem. 2009, 81 (8), 3008-3016. https://doi.org/10.1021/ac802658n.

(18) Skhiri, Y.; Gruner, P.; Semin, B.; Brosseau, Q.; Pekin, D.; Mazutis, L.; Goust, V.; Kleinschmidt, F.; El Harrak, A.; Hutchison, J. B.; et al. Dynamics of Molecular Transport by Surfactants in Emulsions. Soft Matter 2012, 8 (41), 10618. https://doi.org/10.1039/c2sm25934f.

(19) Sandoz, P. A.; Chung, A. J.; Weaver, W. M.; Di Carlo, D. Sugar Additives Improve Signal Fidelity for Implementing Two-Phase Resorufin-Based Enzyme Immunoassays. Langmuir 2014, 30 (23), 6637-6643. https://doi.org/10.1021/la5004484.

(20) Baret, J. C. Surfactants in Droplet-Based Microfluidics. Lab Chip 2011, 12 (3), 422-433. https://doi.org/10.1039/c1lc20582j.

(21) Pan, M.; Rosenfeld, L.; Kim, M.; Xu, M.; Lin, E.; Derda, R.; Tang, S. K. Y. Fluorinated Pickering Emulsions Impede Interfacial Transport and Form Rigid Interface for the Growth of Anchorage-Dependent Cells. ACS Appl. Mater. Interfaces 2014, 6 (23), 21446-21453. https://doi.org/10.1021/am506443e. 
(22) Koudelakova, T.; Chovancova, E.; Brezovsky, J.; Monincova, M.; Fortova, A.; Jarkovsky, J.; Damborsky, J. Substrate Specificity of Haloalkane Dehalogenases. Biochem. J. 2011, 2410 (2), 345-354. https://doi.org/10.1042/BJ20101405.

(23) Iwasaki, I.; Utsumi, S.; Ozawa, T. New Colorimetric Determination of Chloride Using Mercuric Thiocyanate and Ferric Ion. Bull. Chem. Soc. Jpn. 1952, 25 (3), 226. https://doi.org/10.1246/bcsj.25.226.

(24) Scott, R. L. The Solubility of Fluorocarbons. J. Am. Chem. Soc 1948, 70 (12), 4090-4093. https://doi.org/10.1021/ja01192a036.

(25) Gruner, P.; Riechers, B.; Semin, B.; Lim, J.; Johnston, A.; Short, K.; Baret, J. C. Controlling Molecular Transport in Minimal Emulsions. Nat. Commun. 2016, 7, 10392. https://doi.org/10.1038/ncomms10392.

(26) Song, H.; Chen, D. L.; Ismagilov, R. F. Reactions in Droplets in Microfluidic Channels. Angew. Chemie Int. Ed. 2006, 45 (44), 7336-7356. https://doi.org/10.1002/anie.200601554.

(27) Nevolova, S.; Manaskova, E.; Mazurenko, S.; Damborsky, J.; Prokop, Z. Development of Fluorescent Assay for Monitoring of Dehalogenase Activity. Biotechnol. J. 2018 https://doi.org/10.1002/biot.201800144.

(28) Gielen, F.; Butz, M.; Rees, E. J.; Erdelyi, M.; Moschetti, T.; Hyvönen, M.; Edel, J. B.; Kaminski, C. F.; Hollfelder, F. Quantitative Affinity Determination by Fluorescence Anisotropy Measurements of Individual Nanoliter Droplets. Anal. Chem. 2017, 89 (2), 1092-1101. https://doi.org/10.1021/acs.analchem.6b02528.

(29) Nagata, Y.; Hynkova, K.; Damborsky, J.; Takagi, M. Construction and Characterization of Histidine-Tagged Haloalkane Dehalogenase (LinB) of a New Substrate Class from a Gamma-Hexachlorocyclohexane-Degrading Bacterium, Sphingomonas Paucimobilis UT26. Protein Expr. Purif. 1999, 17 (2), 299-304. https://doi.org/10.1006/prep.1999.1143.

(30) Vanacek, P.; Sebestova, E.; Babkova, P.; Bidmanova, S.; Daniel, L.; Dvorak, P.; Stepankova, V.; Chaloupkova, R.; Brezovsky, J.; Prokop, Z.; et al. Exploration of Enzyme Diversity by Integrating Bioinformatics with Expression Analysis and Biochemical
Characterization. ACS Catal. 2018, 8 (3), 2402-2412. https://doi.org/10.1021/acscatal.7b03523.

(31) Koudelakova, T.; Bidmanova, S.; Dvorak, P.; Pavelka, A.; Chaloupkova, R.; Prokop, Z.; Damborsky, J. Haloalkane Dehalogenases: Biotechnological Applications. Biotechnol. J. 2013, 8 (1), 32-45. https://doi.org/10.1002/biot.201100486.

(32) Bednar, D.; Beerens, K.; Sebestova, E.; Bendl, J.; Khare, S.; Chaloupkova, R.; Prokop, Z.; Brezovsky, J.; Baker, D.; Damborsky, J. FireProt: Energy- and Evolution-Based Computational Design of Thermostable Multiple-Point Mutants. PLOS Comput. Biol. 2015, 11 (11), e1004556. https://doi.org/10.1371/journal.pcbi.1004556.

(33) Gruner, P.; Riechers, B.; Semin, B.; Lim, J.; Johnston, A.; Short, K.; Baret, J. C. Controlling Molecular Transport in Minimal Emulsions. Nat. Commun. 2016, 7, 10392. https://doi.org/10.1038/ncomms10392.

(34) Gielen, F.; van Vliet, L.; Koprowski, B. T.; Devenish, S. R. A.; Fischlechner, M.; Edel, J. B.; Niu, X.; DeMello, A. J.; Hollfelder, F. A Fully Unsupervised Compartment-on-Demand Platform for Precise Nanoliter Assays of Time-Dependent Steady-State Enzyme Kinetics and Inhibition. Anal. Chem. 2013, 85 (9), 47614769. https://doi.org/10.1021/ac400480z.

(35) Gielen, F.; Buryska, T.; Vliet, L. Van; Butz, M.; Damborsky, J.; Prokop, Z.; Hollfelder, F. Interfacing Microwells with Nanoliter Compartments: A Sampler Generating High-Resolution Concentration Gradients for Quantitative Biochemical Analyses in Droplets. Anal. Chem. 2014, 87 (1), 624-632. https://doi.org/10.1021/ac503336g.

(36) Yang, T.; Stavrakis, S.; deMello, A. A High-Sensitivity, Integrated Absorbance and Fluorescence Detection Scheme for Probing Picoliter-Volume Droplets in Segmented Flows. Anal. Chem. 2017, 89 (23), 12880-12887. https://doi.org/10.1021/acs.analchem.7b03526.

\section{FOR TOC ONLY}

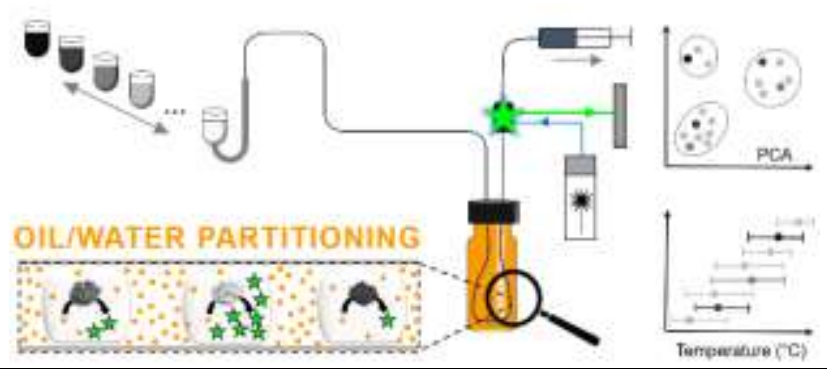

